Paper $136 \quad \begin{aligned} & \text { CCivil-Comp Press, } 2010 \\ & \text { Proceedings of the Seventh International Conference } \\ & \text { on Engineering Computational Technology, } \\ & \text { B.H.V. Topping, J.M. Adam, F.J. Pallarés, } \\ & \text { R. Bru and M.L. Romero, (Editors), } \\ & \text { Civil-Comp Press, Stirlingshire, Scotland }\end{aligned}$

\title{
Failure of Geomaterials Assessed using an Extended Discrete Element Method
}

\author{
C. Ergenzinger, R. Seifried and P. Eberhard \\ Institute of Engineering and Computational Mechanics \\ University of Stuttgart, Germany
}

\begin{abstract}
Strength and failure of geomaterials is investigated using an extended discrete element method (DEM). A rock material is modelled using spherical particles bonded by breakable force elements. An inflation procedure to generate dense sphere packings which is based on the particle's current coordination number is proposed. The particle bonds are enhanced by a progressive failure model that reproduces the effects of singular stress concentrations near crack tips, which are normally not present in DEM, by successive weakening of bonds. The material model is investigated in uni- and triaxial compression, where an efficient approach for simulation of flexible confining is applied, and calibrated to granite yielding wide agreement in strength and failure. A procedure to model shape and angularity of ballast particles is proposed. The strength of ballast stones made from bonded particles is subjected to statistical evaluation and compared to published experiments. Different measures for single particle strength are investigated with respect to the loading state which causes failure.
\end{abstract}

Keywords: discrete element method, bonded particles, failure, strength, geomaterials, strong rock, ballast, particle crushing.

\section{Introduction}

The discrete element method (DEM) can be extended to the simulation of breakable materials by introduction of particle bonds. This approach is particularly advantageous for materials such as rocks, which themselves are granular on micro-scale, since often no adequate continuum descriptions of deformation and especially failure exist. Furthermore, the fracture of bonded particulate matter inherently reproduces many effects of the failure process of this kind of materials.

In this research an implementation of the DEM in the simulation package Pasi- 
modo [1] is used to study the failure of geomaterials, i.e. strong rock and ballast. Firstly, the bonded particle model is described. As dense particle packings are required to produce strong materials, a procedure is proposed to generate sphere packings, that feature a significantly higher average coordination number than standard approaches. Therefore, a particle's current coordination number is used as a control variable for an inflation procedure.

Then, the bonding concept and a progressive failure model based on damage accumulation are introduced. The progressive failure model reduces the strength of the remaining bonds at particles, which have already been involved in bond breakage, in order to promote brittle, localized fracture and reproduce the effects of singular stress concentrations near crack tips that are intrinsically not present in DEM. The material model is calibrated to granite by simulation of uniaxial compression tests. Strength and failure in uniaxial and triaxial compression are investigated in detail. An efficient model of the stress boundary condition in triaxial tests is implemented.

Finally, a procedure to extract realistically shaped angular ballast stones from the granular solid is proposed. Using several tangent planes on ellipsoids of matching size the angular shape of ballast particles can be reproduced reasonably well. This novel approach allows creating breakable, irregularly shaped stones for further simulative investigation. The crushing strength of stones created in this way is statistically evaluated and failure mechanisms and measures of strength are discussed.

\section{Granular Solid from Bonded Particles}

The DEM was developed for the simulation of systems consisting of distinct rock blocks [2]. Its general concept can be applied to any system of particles of arbitrary shape, where the system behaviour is governed by the interaction of these particles on a mesoscopic scale. Usually contact forces are obtained from penalty approaches that avoid particle overlap. Thus, they can be, but do not need to be, based on physical laws. A simple approach to describe the interaction force $F_{i j}$ in normal direction of particles $i$ and $j$ is a linear spring-damper combination

$$
F_{i j}=k_{i j} \delta_{i j}+d \dot{\delta}_{i j}
$$

For spherical particles the overlap is related directly to the particle positions $\delta_{i j}=$ $r_{i}+r_{j}-\left\|\boldsymbol{x}_{i}-\boldsymbol{x}_{j}\right\|$ and the contact force is only applied if $\delta_{i j}>0$.

The stiffness can be calculated based on the idea of an elastic rod (Young's modulus $E)$ between the centres of the particles

$$
k_{i j}=\frac{E A_{i j}}{L_{i j}}=\frac{E \pi r_{i j}^{2}}{2 r_{i j}}=\frac{\pi}{2} E r_{i j}
$$

whose cross section $A_{i j}$ and length $L_{i j}$ depend on the average radius $r_{i j}=\frac{1}{2}\left(r_{i}+r_{j}\right)$. Thus, the idea of an elastic rod introduces some physical motivation into the penalty approach. 
The DEM can be extended to the simulation of granular solids by introducing bilateral particle bonds that generate forces even in case of negative overlap, i.e. a gap, between two bonded particles. A granular solid is generally created by bonding adjacent particles from a dense packing. Fracture and failure phenomena can be incorporated in these models by removal or weakening of bonds based on suitable failure criteria. The advantage of this approach is that multiple fractures at arbitrary locations of the solid may happen and no initiation of cracks is necessary. The crack path is only limited by the discretization, i.e. fracture occurs on the level of bonds between unbreakable particles. For sufficiently small particles this states no serious limitation. Bond breakage based on the loading condition and system dynamics causes cracks to propagate, bifurcate or coagulate.

\subsection{Generation of Dense Sphere Packings}

The generation of a suitable initial configuration is the first step of every DEM simulation. In case of simulations dealing with bonded particles this initial configuration is a dense packing of particles that are to be bonded. A variety of approaches exists for the generation of such a packing, see e.g. [3] and references therein.

A homogeneous and isotropic sphere packing can be obtained by continuously reducing the ratio $\psi=l / 2 \bar{r}$, where $\bar{r}$ denotes the average particle radius and $l$ is a characteristic length of the domain containing the particles. This can be achieved by either compression of the volume containing the particles or inflation of the particles inside of a fixed domain. In both cases a dilute initial configuration of non-overlapping particles has to provided, which can be obtained by using regular lattices that might be randomly disturbed.

Inflation schemes usually employ a radii growth rate $\dot{R}$ which is equal for all particles at one instant in time. In most general form, this scheme reads

$$
\dot{r}(t) / r(t)=\dot{R}(t)
$$

The normalized growth rate $\dot{R}$ might be constant or time dependent.

It is proposed, to modify this scheme by introducing a dependency of a particle's individual normalized growth rate on its coordination number $c_{n}$

$$
\dot{r}(t) / r(t)=\max \left(\dot{R}\left[1-c_{n}(t) / \hat{c}_{n}\right], 0\right),
$$

where $\hat{c}_{n}$ denotes a "desired" coordination number that serves as a further control parameter for the inflation procedure. The intention of this modification is to increase the average coordination number while keeping particle overlap small. As long as the system is dilute, i.e. $\bar{c}_{n}<<1$, the scheme corresponds to Equation (3). When a particle's coordination number rises, this particle's growth rate is reduced. Hence, particles with a smaller coordination number will grow faster than particles with a higher one. This leads to a significantly higher average coordination number. Shrinkage of particles is prohibited, which allows for the use of a desired coordination number $\hat{c}_{n}$ that is 


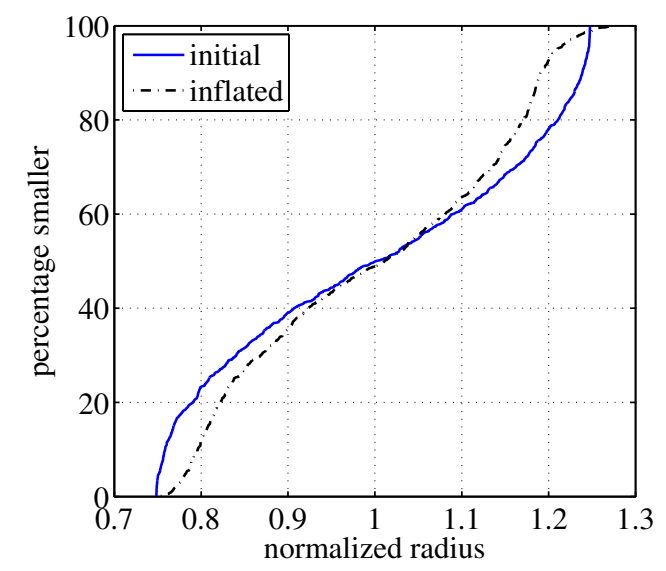

(a) Radii distributions before and after coordination based inflation

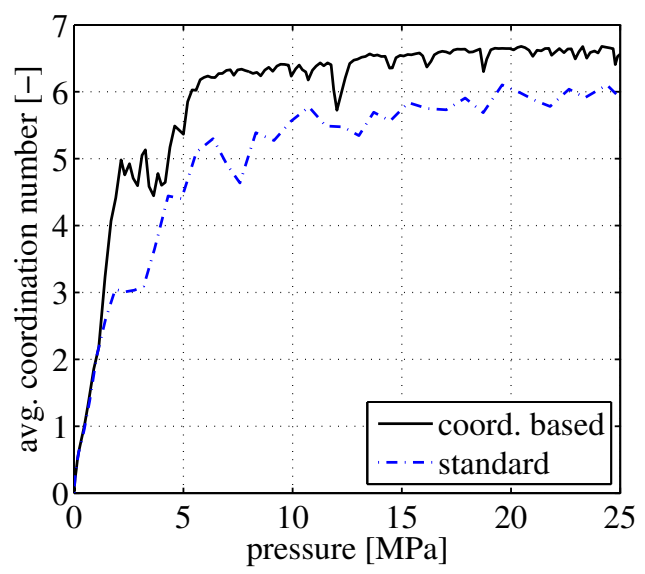

(b) Average coordination number $\bar{c}_{n}$

Figure 1: Standard and coordination number based inflation scheme with $\hat{c}_{n}=8$

smaller than the maximal coordination numbers of all particles. This is important, as the effect of the modified scheme decreases when $\hat{c}_{n}$ is increased. In the limit case of $\hat{c}_{n} \rightarrow \infty$ Equation (4) reduces to Equation (3). On the other hand, if $\hat{c}_{n}$ is chosen too small, no sufficient compaction is achieved.

Obviously, this scheme may lead to a distortion of the particle size distribution. The amount of distortion depends directly on $\hat{c}_{n}$. Smaller $\hat{c}_{n}$ result in a stronger effect on particle size distribution. Usually, the radii distribution is only affected slightly, but clearly, if a well defined radii distribution after compaction is required the scheme cannot be applied. Figure (1a) shows particle size distribution curves before and after coordination based inflation. It can be seen, that the radii of the smaller particles are increased more than those of the larger ones. The larger particles are likely to have a higher number of contacts in an earlier stage of inflation due to their size, whereas the smaller particles may grow with a higher rate. Generally, this trend increases as $\hat{c}_{n}$ is reduced. Nevertheless, the width of the distribution remains nearly unaffected.

The development of the average coordination number is compared in Figure (1b) for the standard and modified scheme. It is apparent that from a pressure above $p \approx 2 \mathrm{MPa}$ the average coordination number is significantly higher with the modified scheme. Final values are $\bar{c}_{n}=6.6$ for the modified and $\bar{c}_{n}=6.0$ for the standard scheme. The latter being the well known number for a random packing of monodisperse spheres. Pressure is used here as a simple measure for stored deformation energy or average overlap of the spheres, as they are directly related by the stiffness of the interactions. Hence, the developed coordination number based inflation scheme yields a sphere packing with a significantly higher average coordination number.

It was realized that during inflation it might be favourable to use soft interactions of the spheres with the boundary walls of the domain for two reasons. Firstly, packings generated with softer walls show a higher average coordination number. Secondly, layering of particles near the walls is reduced, as significant overlap of a number of 
particles and the walls may develop. The effect of particle layering is discussed in [4].

\subsection{Bonding Concept}

Particle bonds usually correspond to force laws which act in restricting at least one degree of freedom of the relative motion of the bonded particles. In this study, the bonds are limited to the normal direction, and are represented by springs that act between the centres of the bonded particles. Shear, bending and twisting is not inhibited $[5,6]$. The model used here employs either a bond or a repulsive contact interaction. The contact force results from the bond as long as it is intact. For particles that are not or no longer bonded a repulsive interaction is applied.

Bonds are created between particles which overlap in the very first time step, i.e. at time $t=0$. The initial length of the spring representing the bond is either based on the positions of the particles to be bonded or on their radii

$$
L_{i j}=\left\{\begin{array}{cc}
\left\|\boldsymbol{x}_{i}-\boldsymbol{x}_{j}\right\| & \text { for IL } 0 \\
r_{i}+r_{j} & \text { for IL } 1,
\end{array}\right.
$$

where IL 0 and IL 1 describe two different model choices. Overlap of bonded particles is defined as the deviation of particle distance from the initial spring length $\delta_{i j}=L_{i j}-\left\|\boldsymbol{x}_{i}-\boldsymbol{x}_{j}\right\|$. Thus, for IL 0 the material is initialized in a relaxed configuration, while for IL 1 internal stresses arise. As the initial overlap should be small compared to the particle radius the initial spring lengths are approximately the same in both cases.

The bond force is calculated as in Equation (1) where now the overlap $\delta_{i j}$ may also become negative. Bond breakage is allowed in tension and compression with different strengths $R_{m, T}$ and $R_{m, C}$, respectively. The maximal forces in a bond are thus

$$
\hat{F}_{i j, \kappa}=R_{m, \kappa} A_{i j}=R_{m, \kappa} \frac{\pi}{4}\left(r_{i}+r_{j}\right)^{2}=R_{m, \kappa} \pi r_{i j}^{2}
$$

where $\kappa=T, C$ indicates the loading state tension and compression, respectively. The breakage force thus depends on the particle radii.

\subsection{Progressive failure model}

As bonded particle models, in contrast to real rock, do not feature singular stress concentrations near crack tips and instable crack propagation [7], a progressive failure model is introduced $[4,8]$. This failure model locally accumulates damage in such a way, that the strength $R_{m}$ of a bond between two particles $i$ and $j$ is calculated according to

$$
R_{m}(t)=\hat{R}_{m}\left(\frac{n_{c, i}(t) n_{c, j}(t)}{n_{c, i,}(0) n_{c, j}(0)}\right)^{a}
$$

where $\hat{R}_{m}$ is the bond strength in undamaged material and $n_{c, \iota}, \iota=i, j$, is the number of a particle's bonds. A similar formulation that has a weaker effect can be obtained 
using the sum instead of the product of the number of bonds. Note that $n_{c, l}(0)$ is the coordination number $c_{n, \iota}(0)$, but generally $n_{c, \iota}(t) \neq c_{n, \iota}(t)$ as the specimen deforms and bonds may break. The exponent $a$ is chosen as the limited, weighted sum of the involved particles' number of broken bonds

$$
a=\min \left(\alpha\left[n_{b, i}(t)+n_{b, j}(t)\right], b\right),
$$

where $\alpha$ is a weighting factor, $b$ denotes a maximal exponent, and the number of broken bonds is $n_{b, \iota}(t)=n_{c, \iota}(0)-n_{c, \iota}(t)$. Thus, breakage of a particle's bonds will successively reduce the strength of the remaining bonds of this particle.

The parameter $\alpha$ adjusts the speed of damage accumulation. Increasing its value will reduce a particle's remaining bonds capability of sustaining loads if few other bonds have been broken. Thus, failure will tend to localize in earlier stages of the damage process. The limitation $b$ controls maximal severity of damage accumulation. If $b$ is chosen too high, complete disintegration may result.

This concept inverts the idea of singular stress peaks, as it reduces the material strength near a point where a stress singularity should appear but cannot due to the particulate nature of the material. Nevertheless, the result is comparable, as in both cases the probability of further damage occurring near this point is increased.

Besides the progressive failure model, particle clusters are introduced in order to generate local heterogeneity, i.e. deformation incompatibilities, while keeping on the large scale homogeneity and isotropy. Bond stiffness and strength of particles in one cluster are chosen differently than for particles that do not belong to the same cluster or that are not part of any cluster. Unbreakable and five times stiffer inter-cluster interactions are applied in this research. Which particles belong to one cluster is determined using a modified three-dimensional generalization of the stamp logic presented in [9] as described in [4]. Here, about one quarter of the particles do not belong to any cluster. Sometimes, the particle clusters are meant to resemble the grains of the material $[9,10]$, but this is not intended here and would not be meaningful due to the chosen particle sizes.

The applicable parameter values of the progressive failure model depend strongly on the presence of clusters. With clustered specimens values of $\alpha=1.5$ and $b=$ 4 turned out to yield a nearly complete, brittle, localized fracture without excessive damage near the process zone.

\section{Strength and Failure Analysis}

The strength of the granular solid created from bonding a dense ensemble of spheres is investigated in uniaxial and triaxial compression. Uniaxial compression serves to adjust Young's modulus $E$ and unconfined compressive strength $C_{0}$ to values reported for granite. Furthermore, the failure process is investigated in detail. Triaxial compression tests reveal if the material model reproduces the dependence of strength and failure modes on the minor principle stresses as observed in experiments. Also, postpeak behaviour and residual strength are investigated. 


\subsection{Failure in Uniaxial Compression}

A specimen is loaded in its axial direction by moving one of its end faces with a constant velocity while the other face is fixed. A strain controlled loading procedure is applied as post-peak behaviour shall be investigated. Instead of using a loading piston to apply forces via an interaction, the boundary layer of particles is fixed to the loading frame. This resembles an infinitely stiff testing machine. Furthermore, load is applied more uniformly as the surface of the sphere packing might be quite rough. Fixing the boundary particles introduces a quite pronounced end constraint, as the particles are completely prevented from moving in lateral direction. It is shown in [4] that the effect of this end constraint is rather small for slender specimens.

The fracturing behaviour of the bonded particle model strongly depends on the width and especially on the shape of the particle size distribution. This is because stiffness and strength in terms of force units depend on the average radius of the bonded particles, see Equations (2) and (6), respectively. It was observed that bond failure seems to concentrate at the large and small particles. Thus, it is expected, that increasing the number of small and large particles will promote failure localization and brittle fracture.

If the initial particle size distribution is chosen according to

$$
r(s) / r_{0}=1+4 a(s-0.5)(1-|s-0.5|),
$$

where $r_{0}$ denotes the average radius, $a$ the distribution half width and $s \in[0,1]$ is a uniformly distributed random number, then the number of particles in a size interval at the limits of the distribution is higher than near the average radius. Indeed, if this particle size distribution with $a=0.25$ is applied, fracture is more brittle and the material behaviour is less plastic than in the case of a uniform distribution [4].

Stress-strain curves of a clustered specimen comprising about 1000 particles with the progressive failure model applied are depicted in Figure (2a). Curves for both cases of Equation (5) are shown. For IL 0 the material is initiated in a relaxed configuration, while internal stresses are present for $I L 1$. The presence of internal stresses reduces the peak strength slightly and the crack initiation stress more noticeably. The strength of $C_{0} \approx 200 \mathrm{MPa}$ is in agreement with values usually reported for granite [11]. In both cases, the fracture propagation is arrested soon after peak. For IL 0 stress is recovered, which is not the case with IL 1 . A nearly complete brittle fracture follows after this short interrupt of the failure process. Stiffness is not affected by internal stresses. The difference in breakage strain as depicted in Figure (2a) is due to the preload of $\sigma(\varepsilon=0) \approx 50 \mathrm{MPa}$ for IL 1, i.e. true breakage strain is about $\varepsilon^{\prime}=0.3 \%$ in both cases. This corresponds to a Young's modulus of $E \approx 65 \mathrm{GPa}$, which is a typical value for granite [11]. A bond Young's modulus of $E_{b}=350 \mathrm{GPa}$ and tensile and compressive bond strengths of $R_{m, T}=845 \mathrm{MPa}$ and $R_{m, C}=2600 \mathrm{MPa}$, respectively, are used in order to obtain these values.

After primary fracture at peak load, stress is recovered up to about $50 \mathrm{MPa}$ and secondary fractures arise. This is due to, firstly, the fact that the specimen is not 


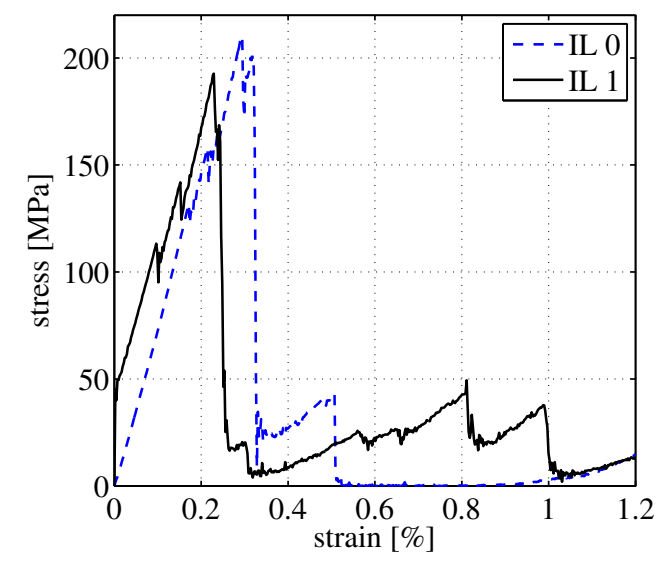

(a) Stress-strain curves with progressive failure model and clusters for different internal stresses (IL 0: initially relaxed, IL 1: with internal stresses)

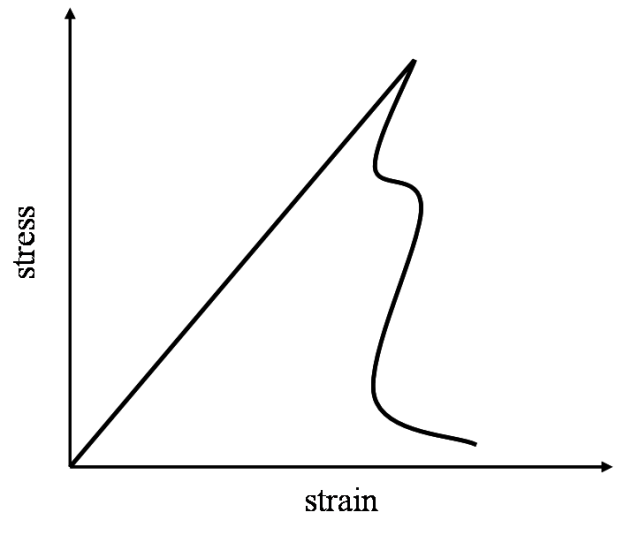

(b) Schematic stress strain curve after $[11,12]$

Figure 2: Stress-strain curves obtained in uniaxial compression from simulations and experiments

broken completely, i.e. a small portion of its cross section remains intact. Secondly, the crack surfaces of the two fragments are pressed against each other for continued loading. This is observed particularly for IL 0 after $\varepsilon \approx 1.0 \%$.

A schematic sketch of a complete stress-strain curve for granite according to experiments in $[11,12]$ is depicted in Figure $(2 b)$. So called class II behaviour, i.e. a stress reversal after peak load, is commonly observed for granite. Yet, at about 60 to $80 \%$ of peak stress strain starts to increase again. Using 25 samples [12], this was found in experiments to happen in a very narrow range, and a second class II type strain reversal was observed as strain had recovered to approximately breakage strain. In strain controlled loading, which is applied in this research, the post-peak curve can drop at most vertically, i.e. a strain reversal is not possible. Thus, it seems reasonable to assume that (i) the true post-peak curve of the discrete element model is class II and (ii) the arrested cracking after $20 \%$ stress release corresponds to the experimentally observed post-peak strain increase. Investigation of the post-peak behaviour using e.g. lateral strain control [13] will be subject of further research.

Analysis of the bond forces reveals that vertically oriented compressive force chains develop, which are connected by horizontal tensile links [4]. Tensile stresses are generated in a compressively loaded specimen due to the heterogeneous nature of the particle model on a meso scale. Maximal compressive bond stresses are about twice the tensile ones. As the ratio of compressive to tensile bond strength is $R_{m, C} / R_{m, T} \approx 3$, first bond breakage is always on tensile loading. Breakage of a horizontally oriented bond on tensile loading corresponds to the formation of a vertical, dilatational microcrack. This is consistent with experimental results [7, 14], where it is found that initial cracking is parallel to the direction of maximum compression and extensile. 
Compressive bond failure always coincides with one or more immediately previous tensile failures of other bonds of the involved particles. The progressive failure model reduces the bond strength at particles, which have been involved in previous bond failure to values that are eventually smaller than the compressive stresses. Thus, tensile failure is the primary mode of damage, as in experiments [7], whereas compressive failure is a means of promoting fracture propagation and localization.

For tensile loading, the described mechanisms are not activated as the tensile bond stresses are higher than the compressive stresses while the compressive bond strength is higher than the tensile strength [4]. This contributes also to explain why tensile strength is usually overestimated in Discrete Element models, see [7].

\subsection{Triaxial Strength and Failure}

The strength of rock depends strongly on the loading state, i.e. the presence and magnitude of minor principle stresses. In experiments cylindrical samples are enclosed in a rubber membrane and hydraulically pressurized, such that $\sigma_{2}=\sigma_{3}<\sigma_{1}$ [15].

\subsubsection{Flexible Confining in DEM}

Different approaches have been proposed to model the boundary conditions in DEM simulations of triaxial tests, see e.g. [16] and references therein. In the simpler approach one rigid wall is pressed against each sample face [10, 17]. This prohibits localization of dilation and thus might increase confining pressure near failure zones resulting in an overestimation of the confined compressive strength and different post peak behaviour. Strain localization is permitted if the lateral stress is applied using a layer of membrane particles that are individually pressurized $[7,16,18]$. The membrane particles can be part of the specimen or inserted around the specimen. The former approaches differ furthermore in identification of the boundary particles and calculation of the corresponding external forces.

The approach proposed here unites the implementational simplicity of the rigid walls with the permission of strain localization of particle membranes. The specimen is enclosed in a membrane of small rigid pressure plates that are individually loaded and free to move independently, see Figure (3a). Thus, the difficulties in calculating corresponding forces on particles forming the membrane can be avoided, while strain may still localize.

The side length of the pressure plates is about 2.5 particle diameters. It is found that further reduction of plate size will not change the material response even well postpeak. A point mass is attached to each of the pressure plates in order to permit their individual movement. For numerical reasons, this mass is chosen to be five times the average particle mass, i.e. approximately the mass of the covered boundary particles.

The stiffness of the interaction between particles and pressure plates is chosen as low as possible in order to achieve a further stress homogenization and avoid loading of single protruding particles. Therefore, the stiffness is calculated proportional to the 


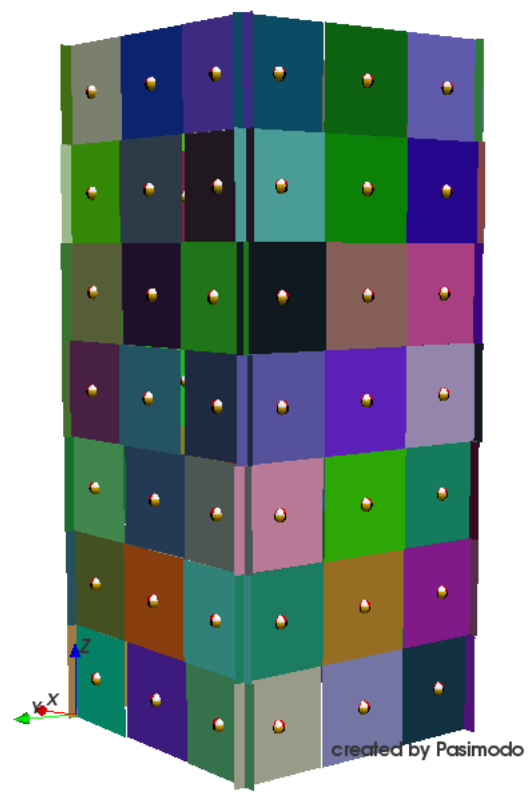

(a) Confining pressure system. A point mass is attached to each pressure plate, which is individually loaded and free to move independent from the others.

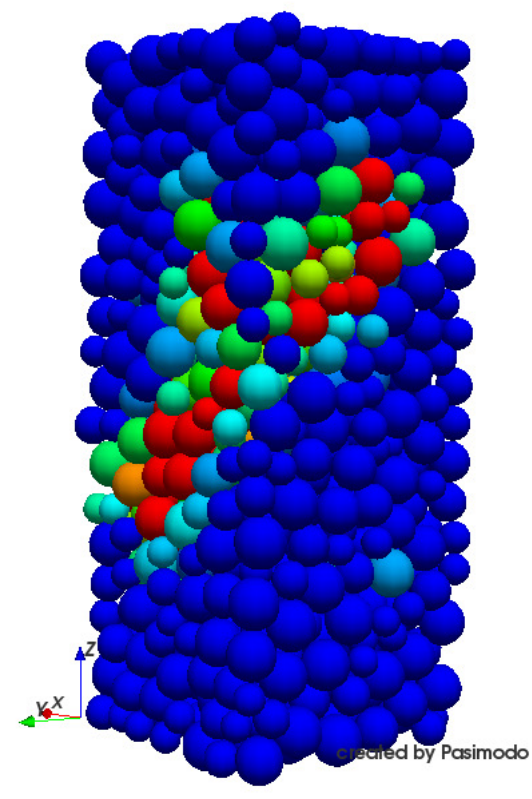

(b) Fractured specimen. Relative number of broken bonds color coded. Blue means all bonds intact, red particles are completely disintegrated.

Figure 3: Triaxial compression test: confining sleeve and fractured specimen

applied confining pressure. Thus, the average overlap of boundary particles and the membrane plates is independent of the applied pressure.

\subsubsection{Simulation Results}

A resultant fracture in confined compression is depicted in Figure (3b). An inclined fracture plane develops, which corresponds to the experimentally observed shear fractures [11] and loci of acoustic emission as reported in [19]. Vertical branches of the major fracture plane indicate initial splitting parallel to the direction of maximum compression as in uniaxial compression simulations and reported from experiments [20]. In many simulations, first fracture localizations are near the sample surface, while initial cracking is distributed through the specimen. This is in agreement with experimental results [19,21]. Finally an inclined fracture zone develops, where some particles are completely disintegrated from the sample.

The development of lateral strain during the failure process is illustrated in Figure (4) for the right and centre column of pressure plates acting on the right face of the specimen in Figure (3b). As long as the specimen is intact, lateral strain is distributed quite homogeneously over its height. After macroscopic fracture at $\varepsilon^{\prime}=0.35 \%$ lateral strain increases strongly and localizes near the fracture plane. Application of the confining pressure with one rigid plate per face would inhibit this localization and 


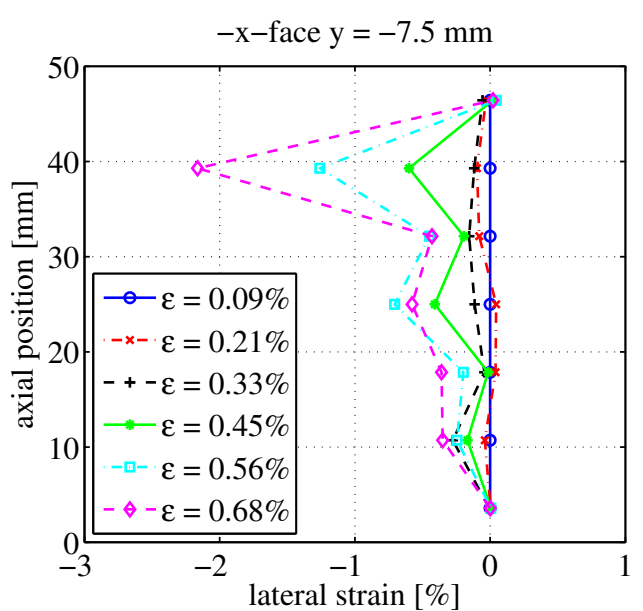

(a) Right face, right column

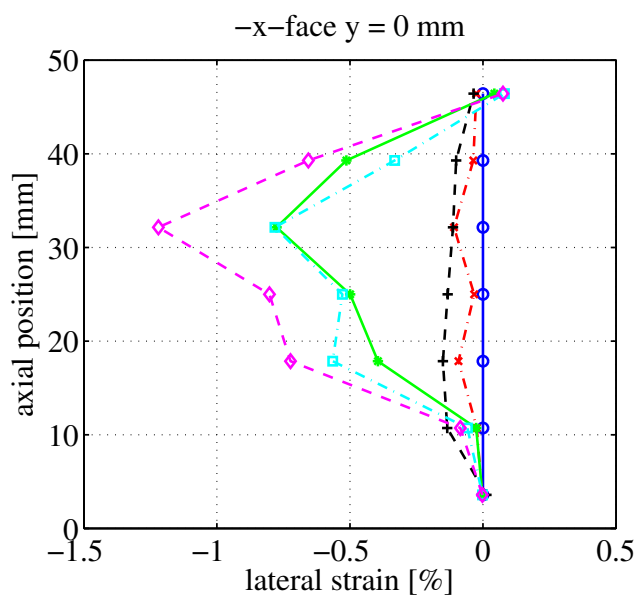

(b) Right face, centre column

Figure 4: Localization of dilation during failure

\begin{tabular}{|c|c|c|c|}
\hline confinement $\sigma_{3}$ & granite [11] & here & PFC3D [10] \\
\hline $1 \mathrm{MPa}$ & $61^{\circ}$ & $49^{\circ}$ & - \\
\hline $10 \mathrm{MPa}$ & $58^{\circ}$ & $31^{\circ}$ & $32^{\circ}$ \\
\hline
\end{tabular}

Table 1: Comparison of friction angles $\Phi$

thus result in a locally higher confining pressure. This would affect the failure process due to the inhomogeneous confinement and the prescription of homogeneous lateral strain.

Stress-strain curves and corresponding failure envelops of a series of confined compression tests are depicted in Figure (5). As axial deformation is inhibited by the strain controll the application of the confining pressure causes an increase in axial stress at the very beginning of the test. Thus, actual breakage strain increases with confining pressure, whereas apparent breakage strain is equal for all pressures in Figure (5a).

The slope of the strength envelope in Figure (5b) can be expressed in terms of Mohr-Coulomb friction angles [15]. In Table 1 the friction angles for two rather low confinements are compared to experimental values [11] and results from published 3D DEM simulations [10]. While for $\sigma_{3}=1 \mathrm{MPa}$ the friction angle is rather close to the experimental one, it is significantly lower for $\sigma_{3}=10 \mathrm{MPa}$. A similar value was found in other DEM simulations using bonded spheres. Thus, it might be suspected that a friction angle $\Phi \approx 30^{\circ}$ is an inherent property of bonded spheres DEM as long as the stress state is not explicitly considered for bond strength as in [18].

The post-peak strength can be interpreted as Griffith locus according to [11] or as strength of the broken rock mass [22]. The former explains the repeated cracking on continued loading with slowly decreasing "peak" stresses as especially for $\sigma_{3}=$ $50 \mathrm{MPa}$. The latter provides the magnitude of residual strength as the fragments of the specimen are pressed against each other. Again, for low confinement reasonable 


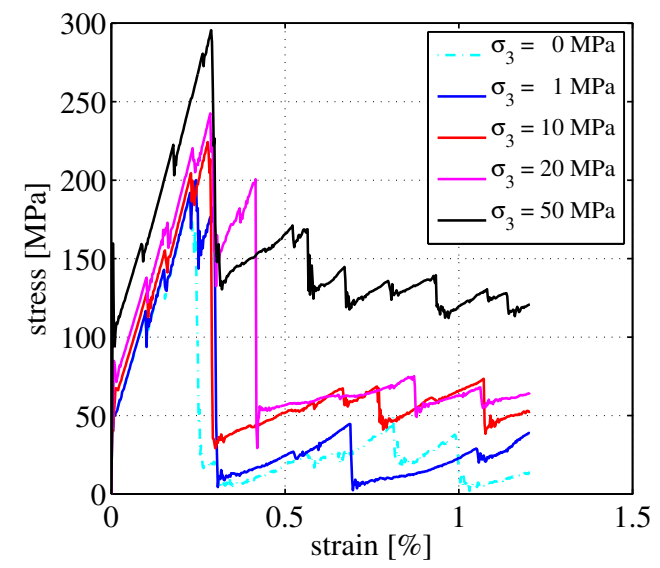

(a) Stress-strain curves for different confining pressures

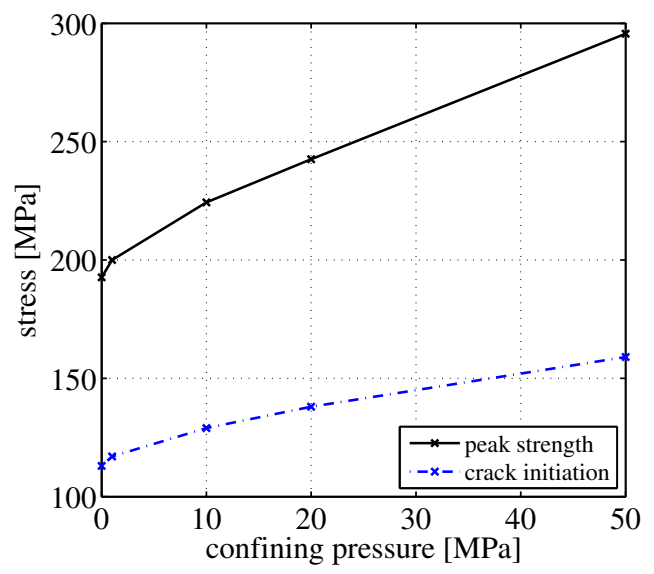

(b) Failure envelopes of the granular solid

Figure 5: Results of a series of confined compression tests

agreement with reported values [22] is found.

In experiments [7] crack initiation was found to occur at approximately constant deviator stresses of about $40 \%$ of the unconfined compressive strength, i.e. $\sigma_{c i}=$ $0.4 C_{0}+\sigma_{3}$. While the magnitude of deviator stress for crack initiation is slightly overestimated in simulations, the dependence on confinement is reproduced quite exactly.

\section{Shape and Strength of Breakable Particle Agglom- erates}

Breakable bonded particle DEM is usually applied to problems related to single agglomerates such as excavations in rock, see e.g. [10]. Applications to media composed of several breakable agglomerates are up to now limited to geometrically simple shapes like disks or spheres [23, 24]. Different approaches to create complex shaped agglomerates have been proposed [25, 26], but they are limited to unbreakable materials as the particle arrangement is chosen according to the agglomerate geometry and not with respect to material behaviour.

It is well known that the strength of an aggregate e.g. of ballast particles depends strongly on the shape and especially angularity of these ballast particles. Furthermore, breakage of ballast particles plays an important role in degradation of ballasted beds. Thus, for numerical simulation a model is required that captures the shape as well as the strength of single ballast particles. A method is proposed here to obtain such agglomerates from a bonded particle DEM material, as described in the previous part of this paper. This allows studying the influence of particle breakage in ballast settlement and degradation. 


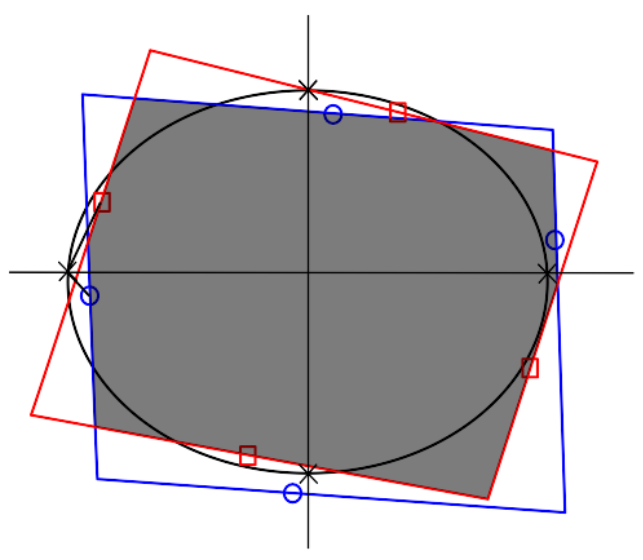

(a) Two-dimensional sketch of the ballast particle shaping approach

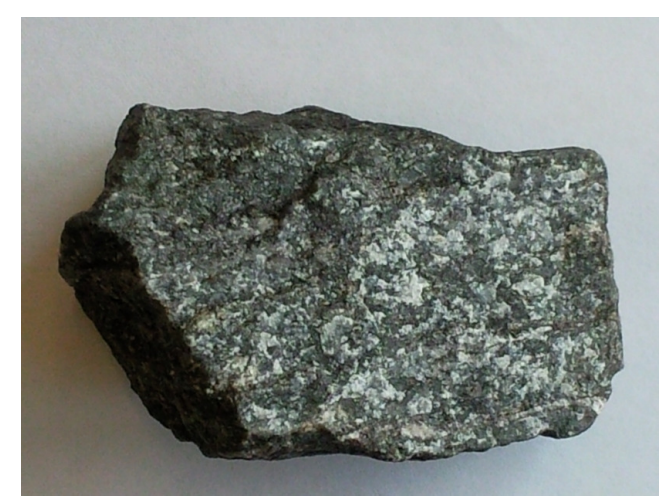

(b) A ballast particle

Figure 6: Comparison of the ballast shaping approach and a real ballast particle

\subsection{Modelling of Ballast Particle Shape}

Single ballast particles are extracted from a large sphere packing by removal of the unnecessary particles. A method to find a mathematical description of the ballast stones is proposed, which is based on tangent planes on ellipsoids. A two-dimensional sketch of this procedure is given in Figure (6a). Starting point for generation of the tangent planes are the intersection points of the ellipsoid with its semiaxes. In order to account for the irregular shape of real stones these intersection points are moved randomly on the surface of the ellipsoid and in normal direction. Finally, tangent planes are constructed in the shifted points and the stone is defined as the volume that is enclosed by all of these planes. It is found that the double application of the procedure with six planes on the same ellipsoid results in realistic shape and angularity. However, the use of more tangent planes at one time would result in more rounded stones as the base points were more uniformly distributed.

This approach is motivated from the finding that ballast stones usually feature quite planar faces, see Figure (6b). Comparison of the sketch and the photo indicates that the described procedure is likely to generate realistically shaped ballast stones. The surface roughness is not explicitly accounted for as in [26], but it is assumed that cutting through an irregular arrangement of spheres will produce a surface whose roughness is comparable to real ballast stones. Two exemplary realizations of stones created with the described approach are depicted in Figure (7). It is apparent that planar faces and sharp edges are formed. The sharpness of the edges is blurred to a small extend by model resolution, i.e. particle size. The projection of the DEM-stones is clearly polygonal and compares well to the general shape of real stones. 


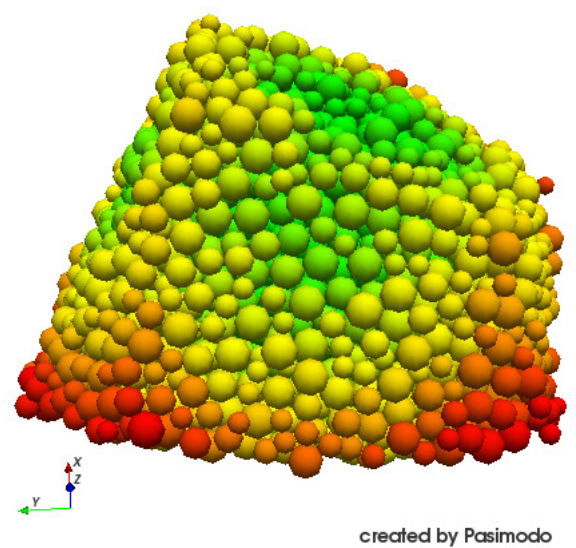

(a) Realization 1

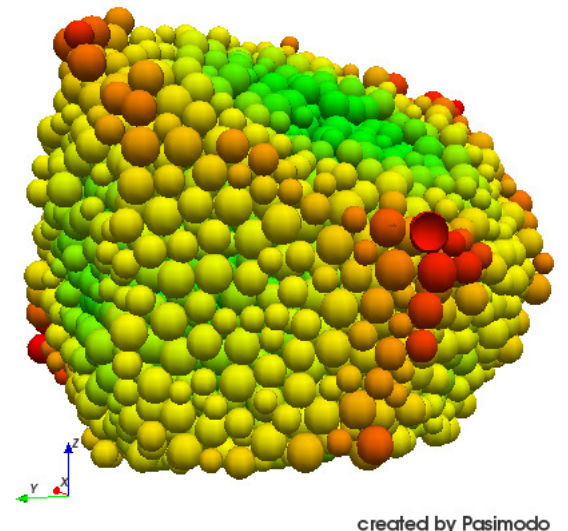

(b) Realization 2

Figure 7: Two realizations of ballast stones. Distance from the origin (located approximately at the center of the stones) color coded in order to clarify the shape.

\subsection{Strength of Ballast Stones}

The strength of single ballast stones whose shape is obtained from the described approach and which consist of granular solid that resembles granite is investigated by compression between parallel platens. An extensive experimental study of the strength of single ballast stones is presented in [27]. The strengths of six types of ballast for various size fractions were investigated and quantified using Weibull statistics. For a given ballast and a given size fraction, the strengths are reported to follow the Weibull distribution reasonably well. A characteristic strength is defined as the ratio of applied force $F$ and the diameter $d$ of the particle [27], i.e. platen distance at failure as

$$
\sigma_{\text {char }}=F / d^{2} \text {. }
$$

For spherical particles loaded with small contact areas this is a measure of the tensile stress acting in the central portion of the particle and it is obtained from elasticity theory [28]. An average strength $\sigma_{0}$ is defined as the value of the characteristic stress such that $1 / e \approx 37 \%$ of the particles survive [27].

The experimental procedure of placing a stone on the lower platen and then lowering the upper one to compress it is adapted to simulative requirements. The manual process of laying the stone down on the lower platen is replaced by gently pressing the lower platen against the stone that is only free to rotate while translational motion is prohibited. Thus, the stone will rotate until a stable configuration is reached. Then, the upper platen is lowered using again a small force to determine the height of the stone, which is not known a priori due to the irregular shape and the procedure of placing the stone on the lower plate. Finally, a strain controlled test is performed.

As a different sphere packing was used for the generation of the stones than for the investigation of material strength and failure, bond parameters are adjusted according 


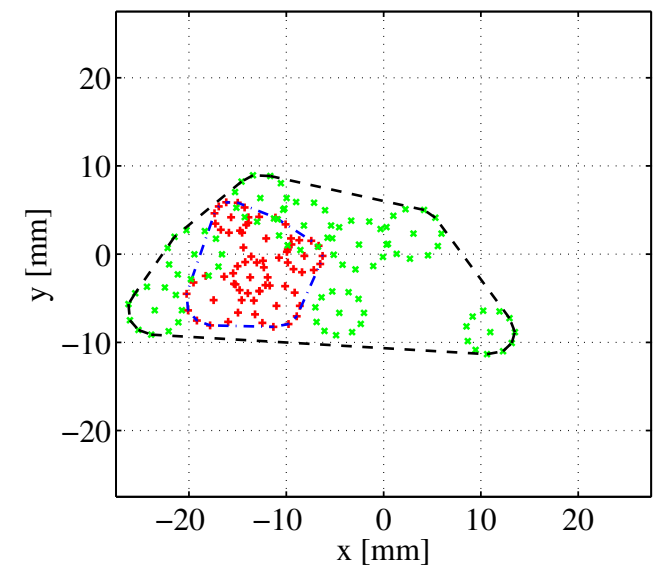

(a) Upper (blue) and lower (black) contact areas

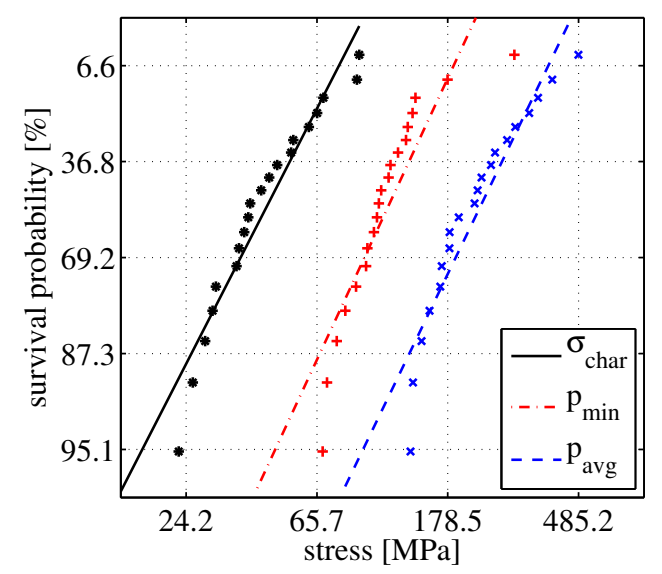

(b) Weibull plots of different measures of strength

Figure 8: Results of single particle crushing tests

to simulations of uniaxial compression tests on a representative sample of 2500 particles from this packing. This gives a bond Young's modulus $E_{b}=700 \mathrm{GPa}$ and tensile and compressive strengths of $R_{m, T}=1300 \mathrm{MPa}$ and $R_{m, C}=4000 \mathrm{MPa}$, respectively.

Unlike in experiments, it is possible to extract the contact area of stone and platens in simulations. The positions of the particles contacting the platens are determined and the smallest polygon enclosing all contact particles is calculated yielding an estimate of the contact area. Therefore, the contact particles themselves are discretized by a number of points, such that they are completely included in the contact area. Figure (8a) shows a typical plot of lower and upper contact areas. Due to the procedure similar to experiments of placing the stone between the platens, the lower contact area is usually larger than the upper one. The average polygonal area is found from simulations to be approximately twice the cross sectional area of all particles in contact with the loading platens. The polygonal area is considered to be more relevant as the stress is distributed to adjacent particles and spacing between contact particles is usually less than two particle diameters. This means that it is assumed that a more homogeneous loading state over an area corresponding to the polygon is obtained slightly below the surface of the stone and that the loading conditions in this region are relevant for bulk fracture. The average contact area is about $600 \mathrm{~mm}^{2}$ for stones comprising at least 500 particles, which would correspond to a contact radius of approximately $14 \mathrm{~mm}$ for a circular contact area. As the diameter of the stones is about $50 \mathrm{~mm}$, the contact area is at the limit of being considered small according to [27, 28].

Four sets of twenty stones comprising different numbers of particles were tested and subjected to a statistical analysis as described in [27]. The strengths $\sigma_{c h a r}$ according to Equation (10) and the contact pressures $p_{\min }$ and $p_{\text {avg }}$ are determined using the larger and the average contact area, respectively. The average contact area is the mean of the large and small contact area of one stone. The corresponding values are sorted in ascending order and for each failure stress a survival probability is calculated 


\begin{tabular}{|r||c|c|c||c|c|c||c|c|c|}
\hline $\mathrm{N}$ & $\sigma_{0}$ & $m_{\sigma}$ & $R_{\sigma}^{2}$ & $p_{\min , 0}$ & $m_{p, \min }$ & $R_{p, \min }^{2}$ & $p_{\text {avg, } 0}$ & $m_{p, \text { avg }}$ & $R_{p, \text { avg }}^{2}$ \\
\hline \hline 300 & 81.45 & 2.43 & 0.97 & 156.83 & 2.32 & 0.93 & 279.14 & 2.85 & 0.97 \\
\hline 500 & 70.38 & 2.20 & 0.92 & 181.30 & 2.59 & 0.90 & 251.24 & 3.41 & 0.98 \\
\hline 700 & 53.36 & 2.66 & 0.93 & 132.71 & 2.93 & 0.81 & 270.16 & 2.82 & 0.90 \\
\hline 1000 & 49.65 & 2.30 & 0.97 & 134.80 & 2.27 & 0.95 & 289.56 & 2.93 & 0.91 \\
\hline
\end{tabular}

Table 2: Statistics of strength. Units of $\sigma_{0}$ and $p_{\min / \text { avg }, 0}$ are MPa.

according to

$$
P_{s}=1-k /(M+1)
$$

where $k$ is the rank of the stone and $M$ the total number of samples. If Weibull statistics applies, a plot of $\ln \left(\ln \left(1 / P_{s}\right)\right)$ against the logarithm of stress yields a straight line, whose slope determines the Weibull modulus $m$, i.e. variability of strength. The data points of a series of tests on stones with about 700 particles and lines of best fit are plotted in Figure (8b). The average strengths and Weibull moduli as well as coefficients of correlation $R^{2}$ for tests on stones comprising different numbers $N$ of particles are given in Table 2. The poor correlation of $p_{\min }$ for $N=700$ is caused by one stone extremely strong in this particular measure. Disregarding this stone, $R_{p, \text { min }}^{2}=0.94$ is obtained.

The results indicate that the behaviour of the DEM stones is not exactly Weibullian, but reasonably close. Especially at lower strengths the data deviates from the Weibull best fit, see Figure (8b). The shape of the plots of all measures of strength is comparable. Generally, a slight curvature to the right of especially $\sigma_{c h a r}$ and $p_{\text {avg }}$, but also $p_{\min }$, is observed. This is in excellent agreement with experimental results presented in [27]. The deviation for lower stresses is interpreted as a minimum strength, below which the probability of failure is zero.

The magnitude of $\sigma_{\text {char }}$ is higher here than reported in experiments on granite, but comparable to small sized granodiorite ballast [27]. Granodiorite is similar to granite in mineralogical composition and usually assumed to be slightly weaker than granite in terms of compressive strength. This might be due to two reasons. Firstly, the material model used here is calibrated to give a compressive strength of $C_{0}=$ $200 \mathrm{MPa}$. But in DEM tensile strength is usually overestimated [7], which is important if the single particle crushing test evaluates in deed tensile strength. In this model Brazilian tensile strength is $T_{B} \approx 20 \mathrm{MPa}$, whereas for granite tensile strength is $T_{0} \approx 10 \mathrm{MPa}$. Furthermore, in [27] no values for compressive strength are reported, so that it is not clear, if the rocks tested there are as strong as assumed here. If tensile failure were identified as the true mode of fracture, calibration could be made with respect to tensile strength.

As mentioned above, the ratio of contact radius to stone diameter is at the limit of what is considered small. Thus, fracture caused by tensile stresses might not be the true mode of failure in these simulations. The values of minimal and average contact pressure are found to be somewhat lower and higher than uniaxial compressive 
strength $C_{0}=200 \mathrm{MPa}$, respectively. Thus, it might be suspected, that compressive strength and failure play a role in single particle crushing. As shown in $[14,20]$, compressive failure in brittle rocks (and the DEM model) is initiated by extensile cleavage parallel to the direction of maximum compression, which makes distinction between compressive and tensile failure awkward.

Compressive bond failure was identified as a means of fracture propagation, see Section 3.1 and [4], which is sensitive to loading conditions. Therefore, the ratio of bonds failing in compression might serve as an indicator of the loading state. The percentage of compressive bond failure is about $30 \%$ for the single particle crushing tests, while it is $40 \%$ in uniaxial compression and $20 \%$ in uniaxial tension, respectively. Thus, it is concluded, that failure of the stones does not occur in purely tensile conditions but that the compressive stress is also important.

Using spherical agglomerates, which consist of substantially more particles, might allow investigating the influence of the contact area on strength and failure modes by variation of platen stiffness. This is not possible for the stones with the given particle numbers as the stone will always bed down on a quite large lower contact area and because loading of single particles at the upper platen would not yield valid results.

The average breakage strain, i.e. the relative difference of platen distance at the beginning and at failure, of stones consisting of at least 500 particles is approximately $4 \%$. In [27] average values of the initial and final platen distance are given. For the investigated granite ballast relative differences of $3.0 \%, 3.4 \%$ and $5.6 \%$ are found for large, medium sized and small ballast, respectively. Slightly higher values are reported for different granodiorite ballasts. The influence of contact stiffness in simulations and contact surface grinding in experiments on these values is not readily accessible. But it is estimated that, at least in simulations, the corresponding uncertainty is not exceeding $0.5 \%$. Thus, the values of simulations compare very well to the experiments. This gives further evidence of the sound calibration of the model and validates the simulative setup for single particle tests.

In quasistatic DEM simulations there is no influence of specimen size on strength unlike in reality, since the specimen can be scaled to any reasonable size without affecting its stiffness and strength [4]. Yet, an influence of particle numbers is present at least for small numbers. Form Table 2 the influence of particle numbers on the different measures of strenght can be evaluated.

The characteristic strength $\sigma_{0}$ clearly decreases with increasing particle numbers $N$ and it seems to converge at $N=1000$. From uniaxial compression tests with varying cross section it is concluded that at least 700 particles per loaded volume element are required to assure discretization independent strength in the present model [4]. These numbers are confirmed by the single stone tests with respect to $\sigma_{0}$. As some of the particles do not contribute to the strength in diametral loading the total number of particles per stone has to be somewhat higher.

The minimum contact pressure $p_{\min , 0}$ shows no clear trend, but a possible effect is weaker than for the characteristic strength $\sigma_{0}$ and it seems that convergence is reached at lower particle numbers. No dependence on particle numbers is detected for $p_{a v g, 0}$, 
but the values fluctuate to some extend. The finding that $p_{a v g, 0}>C_{0}$ is reasonable, since this pressure acts near the loaded surfaces, and will have diminished slightly below surface or in the center, where failure usually initiates. This gives further evidence that compressive failure and strength are important in DEM simulations of single particle crushing.

\section{Conclusion}

A bonded particle model was formulated and successfully calibrated to reproduce strength and failure properties of granite in uni- and triaxial compression. The material results from introduction of breakable bonds between adjacent particles of a dense packing. Highly dense sphere packings are generated with an inflation scheme that uses a particle's coordination number to control its growth rate. Particle bonds that restrict the relative motion in normal direction and may fail in tension and compression are applied. As in bonded particle materials no singular stress concentrations arise [7], a progressive failure model is introduced. The progressive failure model locally accumulates damage and successively reduces the strength of the remaining bonds at particles that have already lost some of their bonds. Thus it promotes localization and allows adjusting brittleness of fracture as the effects of stress concentrations are sort of inverted. Due to this model the failure properties of granite are reproduced. An efficient method to simulate the flexible confining pressure system in triaxial tests was proposed.

A method to extract realistically shaped ballast stones from the granular solid was proposed. With this novel approach it is possible to obtain irregular, angular stones made of breakable material. A number of tangent planes on suitably sized ellipsoids, which are created with a well defined degree of maximal irregularity, is used to define the volume of the stone. Published approaches $[25,26]$ are limited to reproduce the shape of ballast by arranging particles with respect to the generated surface. The presented method can be applied to any given particle setup, which e.g. reproduces the strength of rock. Thus it allows studying degradation processes. The strength of a number of stones shaped with this method and consisting of granular solid is determined by diametral compression. Statistical evaluation reveals excellent qualitative agreement with measurements [27]. Different measures of strength are discussed with respect to the loading conditions that cause failure. It is found that at least in these simulations compressive stresses are important for the failure of the stones.

Consecutive research will address the settling and degradation processes in ballasted track beds using the presented material model and stone shaping approach. Assuming that several hundreds to thousands of stones are required to reliably reproduce the relevant effects total particle numbers will be in the range of one million particles as it was shown that every stone has to comprise at least 700 to 1000 particles. 


\section{Acknowledgements}

This work was partially funded by the German Research Foundation within the Collaborative Research Centre SFB 716 "Dynamic Simulation of Systems with Large Numbers of Particles". Valuable assistance of Celine Geiger and Alexander Tismer in simulative investigations is highly acknowledged. Numerical support and advice of Alexandra Lehnart and Florian Fleißner and discussions with Timo Gaugele are highly appreciated.

\section{References}

[1] F. Fleissner, "Parallel Object Oriented Simulation with Lagrangian Particle Methods", Dissertation, Volume 16 of "Schriften aus dem Institut für Technische und Numerische Mechanik der Universität Stuttgart", Shaker Verlag, Aachen, 2010.

[2] P.A. Cundall, O.D.L. Strack, "A discrete numerical model for granular assemblies", Géotechnique, 29(1), 47-65, 1979.

[3] K. Bagi, "An algorithm to generate random dense arrangements for discrete element simulations of granular assemblies", Granular Matter, 7, 31-43, 2005.

[4] C. Ergenzinger, R. Seifried, P. Eberhard, "A discrete element model to describe failure of strong rock in uniaxial compression”, Granular Matter, (submitted for publication).

[5] F. Fleissner, T. Gaugele, P. Eberhard, "Applications of the discrete element method in mechanical engineering", Multibody System Dynamics, 18(1), 8194, 2007.

[6] T. Gaugele, F. Fleissner, P. Eberhard, "Simulation of material tests using meshfree Lagrangian particle methods", Proceedings of the Institution of Mechanical Engineers, Part K: Journal of Multi-body Dynamics, 222(K4), 327-338, 2008.

[7] M.S. Diederichs, "Instability of Hard Rockmasses: The Role of Tensile Damage and Relaxation", PhD thesis, University of Waterloo, 2000.

[8] C. Ergenzinger, R. Seifried, P. Eberhard, "Modelling of crushable ballast using an extended discrete element method", in "Particle-Based Methods: Fundamentals and Applications", E. Oñate, D.R.J. Owen, (Editors), 134-137, 2009.

[9] N. Cho, C.D. Martin, D.C. Sego, "A clumped particle model for rock", International Journal of Rock Mechanics \& Mining Sciences, 44, 997-1010, 2007.

[10] D.O. Potyondy, P.A. Cundall, "A bonded-particle model for rock", International Journal of Rock Mechanics \& Mining Sciences, 41, 1329-1364, 2004.

[11] C.D. Martin, "The Strength of Massive Lac du Bonnet Granite Around Underground Openings", PhD thesis, University of Manitoba, Winnipeg, Canada, 1993.

[12] W.R. Wawersik, W.F. Brace, "Post-failure behavior of a granite and diabase", Rock Mechanics and Rock Engineering, 3, 61-85, 1971. 
[13] H. Shimizu, T. Koyama, T. Ishida, M. Chijimatsu, T. Fujita, S. Nakama, "Distinct element analysis for class II behavior of rocks under uniaxial compression", International Journal of Rock Mechanics \& Mining Sciences, 4(2), 323-333, 2010.

[14] C. Fairhurst, N.G.W. Cook, "The phenomenon of rock splitting parallel to the direction of maximum compression in the neighbourhood of a surface", in "Proceedings of the First Congress of the International Society of Rock Mechanics", Volume 1, Lisboa, 687-692, 1966.

[15] B.H.G. Brady, E.T. Brown, "Rock Mechanics for Underground Mining", Kluwer Academic Publishers, Dordrecht, 3rd edition, 2004.

[16] G. Cheung, C. O'Sullivan, "Effective simulation of flexible lateral boundaries in two- and three-dimensional DEM simulations", Particuology, 6(6), 483-500, 2008.

[17] Y.P. Cheng, Y. Nakata, M.D. Bolton, "Discrete element simulation of crushable soil”, Géotechnique, 53(7), 633-641, 2003.

[18] Y. Wang, F. Tonon, "Modeling Lac du Bonnet granite using a discrete element model", International Journal of Rock Mechanics \& Mining Sciences, 46(7), 1124-1135, 2009.

[19] B.D. Thompson, P. Young, D.A. Lockner, "Fracture in Westerly granite under AE feedback and constant strain rate loading: nucleation, quasi-static propagation, and the transition to unstable fracture propagation", Pure and Applied Geophysics, 163, 995-1019, 2006.

[20] W.R. Wawersik, C. Fairhurst, "A study of brittle rock fracture in laboratory compression Experiments", International Journal of Rock Mechanics and Mining Sciences \& Geomechanics Abstracts, 7, 561-575, 1970.

[21] D.A. Lockner, J.D. Byerlee, V. Kusenko, A. Ponomarev, A. Sidorin, "Quasistatic fault growth and shear fracture energy in granite", Nature, 350, 39-42, 1991.

[22] E. Hoek, E.T. Brown, "Practical estimates of rock mass strength", International Journal of Rock Mechanics \& Mining Sciences, 34(8), 1165-186, 1997.

[23] W.L. Lim, G.R. McDowell, "Discrete element modelling of railway ballast", Granular Matter, 7, 19-29, 2005.

[24] S. Lobo-Guerrero, L.E. Vallejo, "Discrete element method analysis of railtrack ballast degradation during cyclic loading”, Granular Matter, 8, 195-204, 2006.

[25] M. Lu, G.R. McDowell, "The importance of modelling ballast particle shape in the discrete element method", Granular Matter, 9, 69-80, 2007.

[26] J. Ferellec, G.R. McDowell, "Modelling realistic shape and particle inertia in DEM", Géotechnique, 60(3), 227-232, 2010.

[27] W.L. Lim, G.R. McDowell, A.C. Collop, "The application of Weibull statistics to the strength of railway ballast", Granular Matter, 6, 229-237, 2004.

[28] P.H. Shipway, I.M. Hutchings, "Fracture of brittle spheres under compression and impact loading. I. Elastic stress distributions", Philosophical Magazine A, 67(6), 1389-1404, 1993. 